\title{
Common causes of paediatric alopecia
}

William Cranwell, Rodney Sinclair

This article is the fourth in a series on paediatric health. Articles in this series aim to provide information about diagnosis and management of presentations in infants, toddlers and pre-schoolers in general practice.

\section{Background}

Hair loss in children aged 12 years and younger is most often due to a benign or self-limiting condition. This article presents a review of the assessment of common causes of paediatric alopecia and outlines the implications for general practice.

\section{Objective}

The objective of this article is to help readers systematically assess a child presenting with alopecia, manage the most common diseases of paediatric alopecia and identify patients requiring referral to a dermatologist.

\section{Discussion}

The most common causes of paediatric alopecia are largely non-scarring. These include tinea capitis, alopecia areata, trauma due to traction alopecia or trichotillomania, and telogen effluvium. Scarring alopecia can also occur in childhood and requires scalp biopsy and further investigation by a dermatologist. General practitioners should treat clear cases of tinea capitis. Referral to a dermatologist is necessary in cases when the diagnosis is uncertain, treatment is failing or there is evidence of scarring alopecia.
HAIR LOSS IN CHILDREN aged 12 years and younger encompasses a number of common and rare conditions that may be congenital or acquired. Differentiation of alopecia due to benign causes from that due to serious illness is important for reducing patient and parent distress and offering adequate and prompt diagnosis and treatment. Hair loss disorders are a large, heterogeneous group of conditions that have various clinical features, pathological findings and expected outcomes.

Alopecia in children can be characterised as:

- disorders of hair loss and aberrant hair growth

- hereditary and congenital alopecia

- hair shaft abnormalities

- traumatic alopecia

- infections of the hair. $^{1}$

The most common causes of paediatric alopecia are tinea capitis, alopecia areata, trauma secondary to traction or trichotillomania, and telogen effluvium. ${ }^{2}$ The diagnosis is generally established through directed patient history, scalp and hair examination, trichoscopy and basic laboratory studies. Additional pathological and laboratory investigations may be required after referral to a dermatologist.

Management of paediatric alopecia requires holistic care of the child, parents and any siblings. The clinical manifestation may be subtle or disfiguring and may lead to low selfesteem, depression and social isolation. It is important that parents are given clear information about the expected clinical course and prognosis. Referral to a dermatologist is necessary in cases when the diagnosis is uncertain, treatment is failing or there is evidence of scarring alopecia.

\section{Causes}

The causes of paediatric alopecia include many common and uncommon conditions and syndromes. Alopecia may be due to congenital or acquired conditions. The most common causes of paediatric alopecia seen in general practice are listed in Table 1 . This article will discuss the diagnosis and management of these conditions. Scarring alopecia and hair shaft abnormalities are less common and require further investigation by a dermatologist.

\section{Epidemiology}

Tinea capitis is a common condition to which prepubertal children are predisposed (Figure 1A). ${ }^{3-5}$ The prevalence of positive fungal cultures in children is estimated to be $4-13 \% .^{2,6}$

The point prevalence of alopecia areata is approximately 1 in 1000 people, with a lifetime risk of approximately $2 \%$ (Figure 1B). ${ }^{7,8}$ Most cases occur before age 30 years. Males and females are affected equally.

The prevalence of traction alopecia and trichotillomania is not easily estimated because of underdiagnosis and secretive behaviours. One study of a college student population estimated a lifetime prevalence of trichotillomania of $0.6 \% .{ }^{9}$ Hair loss secondary to pulling and plucking, but not satisfying the Diagnostic and Statistical Manual of Mental Disorders criteria, was reported in $1.5 \%$ of males and $3.4 \%$ of females surveyed. ${ }^{9}$

Acute telogen effluvium may occur at any age, including infants and children. ${ }^{10}$ A study investigating causes of paediatric alopecia found that $2.7 \%$ of children presented with acute telogen effluvium. ${ }^{11}$ Chronic telogen effluvium is less common, typically affecting women aged $30-60$ years. $^{12}$

\section{Assessment and diagnosis}

The ability to differentiate children with easily managed causes of alopecia from those requiring referral and intensive management is an important skill for the general practitioner. 


\section{History}

Children and their parents most often present with complaints of increased hair shedding or patterns of hair loss. A systematic and thorough history will aid diagnosis (Table 2). It is crucial to differentiate between hair shedding and hair breakage.

\section{Examination}

Assessment of a child with alopecia involves examination of the scalp, hair and other body sites. ${ }^{13}$ Examination of the hair and scalp is best performed from above, with adequate lighting. Examine the scalp for evidence of erythema, scales, pustules or papules, erosions and excoriation. These findings may be associated with alopecia or signs of a concomitant scalp disorder (eg seborrhoeic dermatitis or folliculitis). The lack of pinpoint openings (follicular ostia) on the scalp, associated with pustules and ulceration, suggests a scarring alopecia. A kerion is an abscess caused by fungal infection and is characterised by a painful, boggy, inflammatory mass from which any remaining hairs can be pulled out painlessly. ${ }^{1}$

Examination of the hair begins with visual inspection of distribution and density over the scalp. Identify the pattern of hair loss to narrow the differential diagnosis. Hair density is best examined by parting the hair with combs and measuring the distance between the parts. ${ }^{13}$ The hair shafts are examined for length, calibre, fragility and texture. Broken and rough hairs may suggest a disorder of the hair shaft or traumatic alopecia.

Dermoscopy can aid the diagnosis of alopecia in children. Table 3 outlines typical dermoscopic findings that are associated with certain conditions. ${ }^{14}$

A hair pull test identifies active hair shedding and should be performed on

Table 1. Common causes of paediatric alopecia

\begin{tabular}{lll}
\hline Condition & Clinical presentation & Distribution \\
\hline Tinea capitis & Most commonly scaly patches of alopecia or patches & Single or multiple scaly patches with alopecia: patches \\
& of alopecia with small black dots. & enlarge centrifugally over weeks to months. \\
& Pruritus is common. & Patches of alopecia with black dots: black dots are broken \\
& Cervical and occipital lymphadenopathy may be seen & hallicles. \\
in inflammatory cases. & Widespread scaling of the scalp with subtle hair loss. \\
& Children may be asymptomatic carriers. & Kerion: an inflammatory plaque with pustules, crusting and \\
& Dermoscopic features include broken hairs, comma hairs & sinus drainage. ${ }^{29}$ Tender and painful. Potential for scarring. \\
& & Favus: infection with Trichophyton schoenleinii, perifollicular \\
& erythema and cup-shaped yellow crusts. ${ }^{30}$ May progress to & scarring alopecia.
\end{tabular}

Alopecia areata Patchy or confluent hair loss occurring on the scalp or any hair-bearing area of the body.

Typically a circular patch with normal-appearing scalp skin. Dermoscopic features include exclamation point hairs, yellow dots and black dots.

Correlation with atopic dermatitis, hypothyroidism, vitiligo. Nail changes (especially pitting and ridging) are common.

Patchy alopecia areata: most common form, with oval and round patches. ${ }^{1}$

Reticular alopecia areata: irregular pattern in a net-like fashion (reticular).

Ophiasis alopecia areata: band-like pattern of hair loss, most commonly on the temporal or occipital regions.' Poor prognostic feature.

Diffuse alopecia areata: generalised reduction in hair density over the entire scalp. ${ }^{1}$

Alopecia totalis: complete absence of hair on the scalp. Alopecia universalis: complete absence of hair on the scalp and the entire body, including eyebrows, eyelashes, underarms and pubic hair.'

Traction alopecia Due to constant tension on the hair due to styling, ponytails, braiding, use of hair rollers and weaving ${ }^{1,31}$

Fringe sign: retention hair along the frontotemporal hairline. Long-standing, may cause scarring when chronic.

Depends on hair care practice and use of hair products. Most commonly presents with frontotemporal hair loss. May present with patchy hair loss over the scalp in no specific pattern of distribution.

Unusual pattern of hair loss, most commonly affecting the scalp and eyebrows.

Patchy and non-confluent.

May spare peripheral hairs ('Friar Tuck sign'/tonsure pattern).

May present in childhood due to habit or in adolescence as sign of underlying psychological issues. ${ }^{1,32}$ May be associated with other self-harm.

Telogen effluvium Occurs approximately three months after an inciting event (eg medical illness, stress, medication, nutritional disorder). Shedding generally resolves within three to six months, then may take six months for density to improve.

Chronic telogen effluvium if shedding beyond six months.
Diffuse decreased hair density, often characterised by decreased density of ponytail.1.15

Increased hair shedding.

Rarely patchy, unless concomitant patchy alopecia is present. 

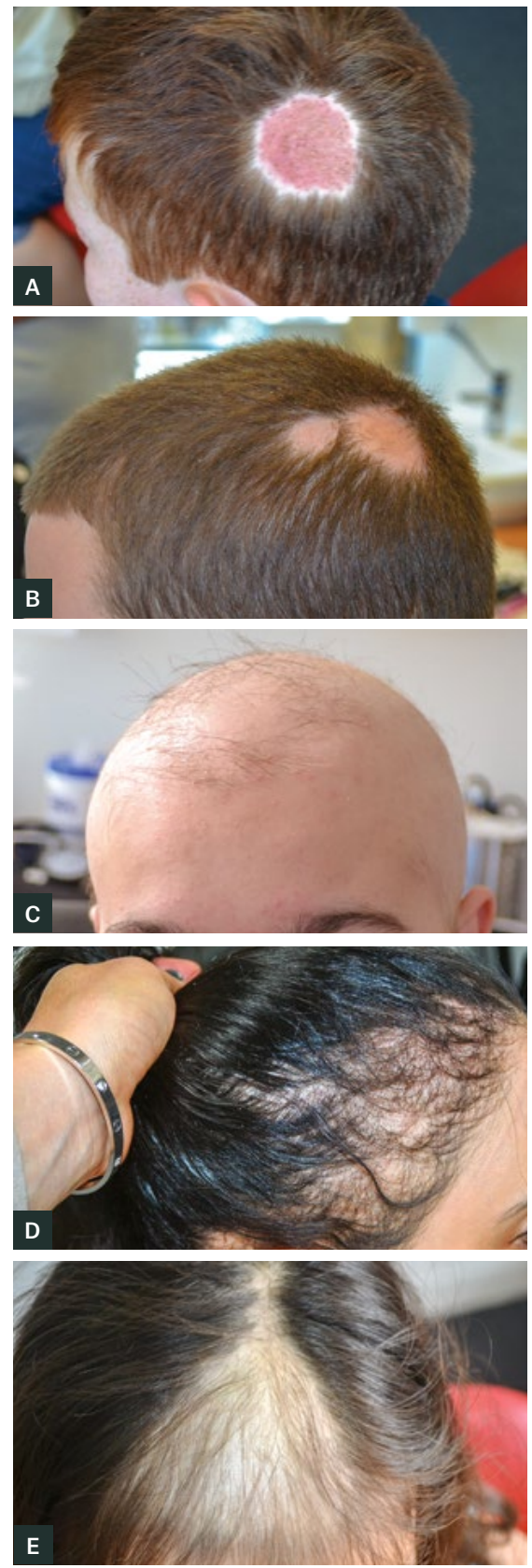

Figure 1. Examples of paediatric alopecia.

A. Tinea capitis presenting with a solitary circular area of hair loss. Note there is a short stubble of broken hairs and the skin is inflamed.

B. Two circumscribed circular areas of alopecia areata. Note the area is completely bald and the skin is normal.

C. Severe alopecia areata. Note a small number of remaining terminal hairs. These would generally also be lost over ensuing months.

D. Traction alopecia caused by repeatedly pulling the hair tightly back into a pony tail over many months.

E. Trichotillomania producing an area of diffuse thinning. Within the area there are numerous broken hairs. The borders are angular. all patients presenting with alopecia. Approximately 50 hairs are grasped at the skin surface and consistent pressure is applied from the proximal to distal ends. The easy extraction of more than six hair fibres suggests increased hair shedding. A specialist may examine the proximal ends of the hairs to identify the predominant hair cycle and characteristics.

Examine other hair-bearing areas to determine the distribution of hair loss. Additional hair, skin, nail and mucosal abnormalities may be present, depending on the condition.

\section{Investigation}

In the majority of cases, scalp biopsy is unnecessary and is traumatic for the child. The diagnosis of alopecia areata, telogen effluvium, traction alopecia and hair shaft abnormalities often does not require investigation. If a scalp biopsy is considered, refer to a dermatologist for further assessment and management. In cases of suspected tinea capitis, scalp scrapings and six to eight hairs should be taken from the affected scalp for fungal microscopy and culture to confirm the diagnosis. ${ }^{1}$ Do not await the results of fungal cultures prior to commencing appropriate systemic therapy. Repeat fungal cultures may be performed after four weeks of treatment.

\section{Management}

The general management of alopecia in children includes managing the underlying cause, providing support and reassurance for the child and parents, camouflage and other cosmetic measures, and psychological support. The psychological effects of hair loss in children can be profound, leading to social

\section{Table 2. Patient history}

\begin{tabular}{|c|c|}
\hline History & Significance \\
\hline $\begin{array}{l}\text { Duration and rate of } \\
\text { hair loss }\end{array}$ & $\begin{array}{l}\text { The duration and rate of hair loss helps differentiate congenital (from } \\
\text { a young age) and acquired (due to an inciting factor or behaviour). This } \\
\text { also determines acute, chronic or transient conditions. }\end{array}$ \\
\hline Location of hair loss & $\begin{array}{l}\text { Determine whether the alopecia is focal, diffuse or patterned. Determine, } \\
\text { in conjunction with physical examination, whether other hair-bearing } \\
\text { body areas are involved. }\end{array}$ \\
\hline Extent of hair loss & $\begin{array}{l}\text { A degree of hair shedding is normal, with normal hair loss of } \\
50-150 \text { hairs per day. }{ }^{33} \text { The use of a hair shedding assessment chart } \\
\text { quantifies hair shedding and allows for objective assessment of } \\
\text { improvement. Determine whether patients or parents have noticed } \\
\text { reduction in ponytail density, although this may only be noticeable after } \\
30 \% \text { decrease in density. }{ }^{34}\end{array}$ \\
\hline Associated symptoms & $\begin{array}{l}\text { The presence of associated symptoms, including pain, tenderness, } \\
\text { pruritus and burning sensation, are associated with certain diagnoses. } \\
\text { Symptoms may be present due to concomitant diseases (eg seborrhoeic } \\
\text { dermatitis). }\end{array}$ \\
\hline $\begin{array}{l}\text { Differentiation of } \\
\text { hair loss versus hair } \\
\text { breakage }\end{array}$ & $\begin{array}{l}\text { Determining true hair shedding versus hair breakage helps differentiate } \\
\text { causes of alopecia from hair shaft disorders or traumatic causes of } \\
\text { alopecia. Enquire about the presence of pain when removing hairs } \\
\text { (painless extraction of hairs from the scalp is characteristic of loose } \\
\text { anagen hair syndrome). }\end{array}$ \\
\hline Hair care behaviour & $\begin{array}{l}\text { The use of hair care products and grooming behaviour is important for } \\
\text { diagnosing traction alopecia or hair care that damages the hair shaft } \\
\text { (eg use of chemicals). }\end{array}$ \\
\hline $\begin{array}{l}\text { Medical and family } \\
\text { history }\end{array}$ & $\begin{array}{l}\text { Questions about past medical history and family history of alopecia } \\
\text { (often undiagnosed) may assist diagnosis. In adolescent females, } \\
\text { enquire about menarche. A diagnosis of telogen effluvium is often made } \\
\text { when an inciting factor is identified (eg medical illnesses, stress, poor } \\
\text { diet, medications). }\end{array}$ \\
\hline
\end{tabular}


isolation, low self-esteem, depression and humiliation. ${ }^{15,16}$ Consider referral to a child and adolescent psychologist or psychiatrist if appropriate. Children with extensive hair loss may require a wig, hairpiece or false eyelashes.

Treatment of tinea capitis must begin once the clinical diagnosis is made, without awaiting fungal culture results. Oral antifungal treatment is required for tinea capitis, as topical antifungal treatment has inadequate penetration into the hair follicles. Oral griseofulvin is the first-line therapy on the basis of efficacy in randomised control trials. ${ }^{17}$ The typical starting dose is $20-25 \mathrm{mg} / \mathrm{kg} /$ day for six to 12 weeks. ${ }^{18}$ Terbinafine is an alternative first-line agent, with data suggesting it is at least as effective as griseofulvin. ${ }^{17} \mathrm{~A}$ high incidence of tinea capitis is found among Aboriginal and Torres Strait Islander children, with Trichophyton tonsurans often implicated. This organism is more sensitive to systemic terbinafine, and resistance to griseofulvin is common. Systemic antifungal therapy is generally well tolerated, with gastrointestinal distress, headache and skin eruptions the most common side effects. Laboratory investigations and monitoring are not required unless treatment extends beyond eight weeks. If a prolonged course is required, monitor liver and renal function for toxicity. Treatment of kerion and favus require referral to a dermatologist for systemic glucocorticoids and close monitoring. The prognosis for children with tinea capitis is excellent, with complete clearance seen in most patients who are adequately treated. Failure to identify the diagnosis or prolonged infection confers the greatest risk of irreversible alopecia.

Family members and close contacts should be examined for tinea capitis and should be treated simultaneously if detected. Given the risk of asymptomatic carrier status, family members should use antifungal hair shampoo for two to four weeks and avoid sharing hair products or other equipment (eg helmets or hats). Pets may be reservoirs for dermatophytes, so assessment by vets is advised if multiple members of a household are affected.

Therapeutic options for alopecia areata in children are limited because of concerns about treatment tolerability. Referral of children with alopecia areata to a dermatologist is appropriate (Figure 1C). Intralesional glucocorticoids are often used, but low tolerability as a result of pain and anxiety during injections is a limiting factor. ${ }^{19}$ Potent topical glucocorticoids are the first-line treatment. ${ }^{1,20,21}$ Topical minoxidil and topical immunotherapy are also treatment options..$^{22}$ The use of systemic glucocorticoids may induce hair growth, but children most often relapse on cessation of treatment. ${ }^{23}$ Long-term use of glucocorticoids is not indicated

\section{Table 3. Dermoscopic findings of alopecia}

\begin{tabular}{ll}
\hline Finding & Associated condition \\
\hline Absence of follicular ostia & Destruction of follicle opening due to scarring alopecia \\
\hline Black dots & Fibrosis associated with scarring alopecia \\
\hline Yellow dots & $\begin{array}{l}\text { Broken hairs at the scalp surface - alopecia areata, } \\
\text { tinea capitis }\end{array}$ \\
\hline Exclamation points & Accumulation of sebum and keratin - alopecia areata \\
\hline Comma hairs & $\begin{array}{l}\text { Associated with alopecia areata and trichotillomania } \\
\text { Medical and family history }\end{array}$ \\
$\begin{array}{l}\text { Associated with tinea capitis } \\
\text { Questions about past medical history and family history } \\
\text { of alopecia (often undiagnosed) may assist diagnosis. } \\
\text { In adolescent females, enquire about menarche. A } \\
\text { diagnosis of telogen effluvium is often made when an } \\
\text { inciting factor is identified (eg medical illnesses, stress, } \\
\text { poor diet, medications). }\end{array}$ \\
\hline
\end{tabular}

in children. Treatment with ultraviolet therapy has seen variable results. ${ }^{21}$

Traction alopecia may be reversible if identified and if the hairstyle or behaviour is modified (Figure 1D). Prolonged traction on the hair may lead to irreversible scarring. ${ }^{1}$ Childhood trichotillomania is often a benign inadvertent behaviour that children may outgrow (Figure 1E). Counselling the patient and parents about the behaviour and modifications can occasionally be successful. ${ }^{1}$

Management of adolescent trichotillomania is more difficult and may represent underlying psychological distress. Adolescents are often more secretive with behaviours, and the diagnosis is difficult to determine. Psychological therapy and counselling may identify the underlying problem and modify behaviour ${ }^{1}$. Referral to a psychiatrist for recalcitrant cases is advised. ${ }^{24}$ When pharmacotherapy is selected for treatment, serotonin reuptake inhibitor antidepressants may be effective in treating obsessivecompulsive disorder. ${ }^{25} \mathrm{~N}$-acetylcysteine has been used alone or in combination with antidepressants for treatment of obsessive-compulsive disorder with good effect. ${ }^{26,27}$

Given that telogen effluvium is generally a reactive and self-limiting condition, few treatment options exist. ${ }^{28}$ Treatment of telogen effluvium is generally reassurance and avoidance of triggers. ${ }^{1}$ Most patients are reassured that complete baldness is not possible (unless concomitant hair disorder exists), telogen effluvium is temporary and regrowth is likely. The management approach for telogen effluvium includes identification and removal of the inciting factor, camouflaging hair loss and psychological support. The efficacy of topical minoxidil in telogen effluvium is unclear. Theoretically, minoxidil should hasten resolution of hair growth by prolonging anagen and stimulating telogen hairs to re-enter anagen. ${ }^{1,28}$ However, it is not considered first-line therapy.

\section{Indications for referral}

Refer any case of paediatric alopecia to a dermatologist for further assessment and 
treatment if the diagnosis is uncertain or the case is not a typical presentation of a particular condition. Obtaining appropriate basic investigations (eg fungal cultures) in general practice prior to review may expedite diagnosis and treatment. Refer any children requiring scalp biopsy to mitigate the need for repeat biopsies and unnecessary investigations.

Other indications for referral include:

- tinea capitis with severely inflamed scalp skin, evidence of a kerion or failure to respond to treatment (either treatment-resistant or alternative diagnosis)

- alopecia areata, particularly if rapid hair shedding, totalis or universalis are present

- cases requiring intralesional steroid injection and systemic therapy - refer to a dermatologist with experience in alopecia

- suspected telogen effluvium persisting longer than three months

- all suspected cases of scarring alopecia, characterised by alopecia accompanied with papules or pustules, erythematous plaques and atrophy, telangiectasia, follicular hyperkeratosis and ulceration

- these children are likely to require scalp biopsy and further investigations.

\section{Conclusion}

Paediatric alopecia is an uncommon but important presentation in general practice. Alopecia may be focal, diffuse or patterned. A number of conditions cause significant morbidity and contribute to significant psychosocial stress for patients and parents. A systematic approach to assessment, including a thorough history, full-body examination and investigations guided by clinical presentation, leads to accurate diagnosis and early referral to appropriate specialists. Treatment of typical cases of tinea capitis with oral antifungal treatment is indicated in general practice. Consider referral to a dermatologist if the diagnosis is unclear, treatment is failing or there is evidence of scarring alopecia.

\section{Authors}

William Cranwell MBBS (Hons), BMedSc (Hons), MPH\&TM, Clinical Research Fellow, Sinclair Dermatology, East Melbourne, Vic

Rodney Sinclair MBBS, MD, FACD, Director, Sinclair Dermatology, East Melbourne, Vic. rodney.sinclair@ sinclairdermatology.com.au

Competing interests: None.

Provenance and peer review: Commissioned, externally peer reviewed.

\section{References}

1. Harrison S, Sinclair R. Optimal management of hair loss (alopecia) in children. Am J Clin Dermatol 2003;4(11):757-70.

2. Nield LS, Keri JE, Kamat D. Alopecia in the general pediatric clinic: Who to treat, who to refer. Clin Pediatr (Phila) 2006;45(7):605-12.

3. Mirmirani P, Tucker LY. Epidemiologic trends in pediatric tinea capitis: A population-based study from Kaiser Permanente Northern California. J Am Acad Dermatol 2013;69(6):916-21. doi: 10.1016/j. jaad.2013.08.031.

4. Emele FE, Oyeka CA. Tinea capitis among primary school children in Anambra state of Nigeria. Mycoses 2008;51(6):536-41. doi: 10.1111/j.1439-0507.2008.01507.x.

5. Mapelli ET, Cerri A, Bombonato C, Menni S. Tinea capitis in the paediatric population in Milan, Italy: The emergence of Trichophyton violaceum. Mycopathologia 2013;176(3-4):243-46. doi: 10.1007/s11046-013-9637-0.

6. Abdel-Rahman SM, Farrand N, Schuenemann E, et al. The prevalence of infections with Trichophyton tonsurans in schoolchildren: The CAPITIS study. Pediatrics 2010;125(5):966-73. doi: 10.1542/peds.2009-2522.

7. Safavi KH, Muller SA, Suman VJ, Moshell AN, Melton LJ 3rd. Incidence of alopecia areata in Olmsted County, Minnesota, 1975 through 1989. Mayo Clin Proc 1995;70(7):628-33.

8. Madani S, Shapiro J. Alopecia areata update. J Am Acad Dermatol 2000;42(4):549-66.

9. Christenson GA, Pyle RL, Mitchell JE. Estimated lifetime prevalence of trichotillomania in college students. J Clin Psychiatry 1991;52(10):415-17.

10. Atton AV, Tunnessen WW Jr. Alopecia in children: The most common causes. Pediatr Rev 1990;12(1):25-30.

11. Nnoruka EN, Obiagboso I, Maduechesi C. Hair loss in children in South-East Nigeria: Common and uncommon cases. Int J Dermatol 2007;46(s1):18-22.

12. Trüeb RM. Systematic approach to hair loss in women. J Dtsch Dermatol Ges 2010;8(4):284-97. doi: 10.1111/j.1610-0387.2010.07261.x.

13. Jackson AJ, Price VH. How to diagnose hair loss. Dermatol Clin 2013;31(1):21-28. doi: 10.1016/j.det.2012.08.007.

14. Mubki T, Rudnicka L, Olszewska M, Shapiro J. Evaluation and diagnosis of the hair loss patient: Part I. History and clinical examination. J Am Acad Dermatol 2014;71(3):415.e1-e15. doi: 10.1016/j.jaad.2014.04.070.

15. Liakopoulou M, Alifieraki T, Katideniou A, et al. Children with alopecia areata: Psychiatric symptomatology and life events. J Am Acad Child Adolesc Psychiatry 1997;36(5):678-84.

16. Ghanizadeh A. Comorbidity of psychiatric disorders in children and adolescents with alopecia areata in a child and adolescent psychiatry clinical sample. Int J Dermatol 2008;47(11):1118-20. doi: 10.1111/j.13654632.2008.03743.x.

17. Chen $X$, Jiang $X$, Yang $M$, et al. Systemic antifungal therapy for tinea capitis in children. Cochrane Database Syst Rev 2016;(5):CD004685. doi: 10.1002/14651858.CD004685.pub3.

18. Gupta AK, Cooper EA. Update in antifungal therapy of dermatophytosis. Mycopathologia 2008;166(5-6):353-67. doi: 10.1007/s11046-008-9109-0.

19. Madani S, Shapiro J. Alopecia areata update. J Am Acad Dermatol 2000;42(4):549-66.

20. Lenane P, Macarthur C, Parkin PC, et al. Clobetasol propionate, $0.05 \%$, vs hydrocortisone, $1 \%$, for alopecia areata in children: A randomized clinical trial. JAMA Dermatol 2014;150(1):47-50. doi: 10.1001/jamadermatol.2013.5764.

21. Hawit F, Silverberg NB. Alopecia areata in children. Cutis 2008;82(2):104-10.

22. Sinclair R. Aloepeic Areata. In: Williams H, Bigby M, Diepgen T, Herxheimer A, Naldi L, Rzany B, editors. Evidence based dermatology. BMJ Publishing Group. London, 2014.

23. Alabdulkareem AS, Abahussein AA, Okoro A. Severe alopecia areata treated with systemic corticosteroids. Int J Dermatol 1998;37(8):622-24.

24. Sinclair R. Diffuse hair loss. Int J Dermatol 1999;38 Suppl 1:8-18.

25. Soomro GM, Altman DG, Rajagopal S, Oakley-Browne M. Selective serotonin re-uptake inhibitors (SSRIs) versus placebo for obsessive compulsive disorder (OCD). Cochrane Database Syst Rev 2008;(1):CD001765. doi: 10.1002/14651858.CD001765.pub3.

26. Oliver G, Dean O, Camfield D, et al. N-acetyl cysteine in the treatment of obsessive compulsive and related disorders: A systematic review. Clin Psychopharmacol Neurosci 2015;13(1):12-24. doi: 10.9758/cpn.2015.13.1.12.

27. Paydary K, Akamaloo A, Ahmadipour A, Pishgar F, Emamzadehfard S, Akhondzadeh $\mathrm{S}$. $\mathrm{N}$-acetylcysteine augmentation therapy for moderate-to-severe obsessive-compulsive disorder: Randomized, double-blind, placebo-controlled trial. J Clin Pharm Ther 2016;41(2):214-19. doi: 10.1111/jcpt.12370.

28. Harrison S, Sinclair R. Telogen effluvium. Clin Exp Dermatol 2002;27(5):389-95.

29. Isa-Isa R, Arenas R, Isa M. Inflammatory tinea capitis: Kerion, dermatophytic granuloma, and mycetoma. Clin Dermatol 2010;28(2):133-36. doi: 10.1016/j.clindermatol.2009.12.013.

30. Ilkit M. Favus of the scalp: An overview and update. Mycopathologia 2010;170(3):143-54 doi: 10.1007/s11046-010-9312-7.

31. Whiting DA. Traumatic alopecia. Int J Dermatol 1998;38 Suppl 1:34-44.

32. Sinclair RD, Banfield CC, Dawber RPR, editors. Non-scarring traumatic alopecia. In: Handbook of diseases of the hair and scalp. Oxford: Blackwell Science, 1999: p. 85-91.

33. Paus $R$, Cotsarelis $G$. The biology of hair follicles N Engl J Med 1999;341(7):491-97.

34. Moghadam-Kia S, Franks AG Jr. Autoimmune disease and hair loss. Dermatol Clin 2013;31(1): 75-91. doi: 10.1016/j.det.2012.08.008.

correspondence ajgp@racgp.org.au 